Pacific Journal of Mathematics

ON THE SIGN OF GREENS FUNCTIONS FOR MULTIPOINT 


\title{
ON THE SIGN OF GREEN'S FUNCTIONS FOR MULTIPOINT BOUNDARY VALUE PROBLEMS
}

\author{
JERRY RIDENHOUR
}

If an $n$th order linear ordinary differential equation $L y=0$ is disconjugate, then the sign of the Green's functions is well known. In this work, it is only supposed that $L y=0$ is $\left(i_{1}, \cdots, i_{k}\right)$-disconjugate for certain values of $i_{1}, \cdots, i_{k}$, and the sign of the Green's functions for certain multipoint problems is shown to be the same as when $L y=0$ is disconjugate. The results extend earlier ones on two-point problems due to Peterson. The proofs simplify Peterson's arguments in a way such that the analysis of two-point problems is not only easier but carriers over to multipoint problems.

1. Introduction and preliminaries. We concern ourselves with a fixed compact interval $[a, b]$ and real-valued solutions of the $n$th order linear differential equation $L y=0$ where

$$
L y \equiv y^{(n)}+p_{n-1}(t) y^{(n-1)}+\cdots+p_{0}(t) y
$$

and $p_{i} \in C[a, b], i=0, \cdots, n-1$.

For the basic facts about disconjugacy and Green's functions, the reader is referred to Chapter 3 of Coppel's monograph [3]. As usual, $L y=0$ is said to be conjugate on an interval $I$ provided there exists a nontrivial solution of $L y=0$ with at least $n$ zeros counting multiplicities on $I$; in the contrary case, $L y=0$ is said to be disconjugate on $I$. If $L y=0$ is conjugate on the interval $[\alpha, \beta]$, then the first conjugate point of $\alpha$, denoted by $\eta_{1}(\alpha)$, is the infimum of the numbers $t \in(\alpha, \beta]$ such that $L y=0$ is conjugate on $[\alpha, t]$. If $a_{1}, \cdots, a_{k}$ are distinct points in $[a, b]$ and $i_{1}, \cdots, i_{k}$ are nonnegative integers, then a function $f$ defined on $[a, b]$ is said to have $\left(i_{1}, \ldots, i_{k}\right)$ zeros at $\left(a_{1}, \ldots, a_{k}\right)$ if $f$ has $i_{j}$ derivatives at $a_{j}$ and $f^{(l)}\left(a_{j}\right)=0$ for $1 \leqq j \leqq k, 0 \leqq l \leqq i_{j}-1$. If $i_{1}+\cdots+i_{k} \geqq n$, then $L y=0$ is said to be $\left(i_{1}, \cdots, i_{k}\right)$-disconjugate on an interval $I$ provided that no nontrivial solution of $L y=0$ has $\left(i_{1}, \ldots, i_{k}\right)$-zeros at $\left(a_{1}, \ldots, a_{k}\right)$ for any choice of $a_{1}, \cdots, a_{k}$ in $I$ with $a_{1}<\ldots<a_{k}$.

Consider the boundary-value problem (BVP for short)

$$
\left\{\begin{aligned}
L y= & f, y \text { has }\left(i_{1}, \cdots, i_{k}\right) \text {-zeros at }\left(a_{1}, \cdots, a_{k}\right), \\
& i_{1}+\cdots+i_{k}=n, a=a_{1}<\cdots<a_{k}=b,
\end{aligned}\right.
$$

and the polynomial 


$$
P(t) \equiv\left(t-a_{1}\right)^{i_{1}} \cdots\left(t-a_{k}\right)^{i_{k}} .
$$

We will refer to (1.1) as the BVP assigning $\left(i_{1}, \cdots, i_{k}\right)$-zeros at $\left(a_{1}, \cdots, a_{k}\right)$. If no nontrivial solution of $L y=0$ has $\left(i_{1}, \cdots, i_{k}\right)$-zeros at $\left(a_{1}, \cdots, a_{k}\right)$, then (1.1) is uniquely solvable for all $f \in C[a, b]$ and the solution is given by

$$
y(t)=\int_{a}^{b} G(t, s) f(s) d s, \quad t \in[a, b]
$$

where $G(t, s)$ is the Green's function. $G(t, s) \in C([a, b] \times[a, b])$ is uniquely determined by the requirement that, for each fixed $s \in(a, b)$, $g(t) \equiv G(t, s)$ must satisfy the three conditions: (i) $L g=0$ on $a \leqq$ $t<s$ and $s<t \leqq b$, (ii) $g$ has $\left(i_{1}, \ldots, i_{k}\right)$-zeros at $\left(a_{1}, \cdots, a_{k}\right)$, and (iii) $g^{(i)}(s+0)-g^{(i)}(s-0)=0$ or 1 according as $0 \leqq i \leqq n-2$ or $i=n-1$. For convenience, we make the following

Definition. The polynomial $P(t)$ is said to determine the sign of $G(t, s)$ if $P(t) G(t, s) \geqq 0$ for all $(t, s) \in[a, b] \times(a, b)$ with equality only when $t \in\left\{a_{1}, \cdots, a_{k}\right\}$.

In a very important result, Levin (see the reference on p. 46 of [7] to Levin's 1961 doctoral dissertation) and Čičkin [2] showed independently that $P(t)$ determines the sign of $G(t, s)$ when $L y=0$ is disconjugate.

The disconjugacy of $L y=0$ obviously implies $\left(i_{1}, \ldots, i_{k}\right)$-disconjugacy whenever $i_{1}+\cdots+i_{k} \geqq n$; however, it is possible for $L y=0$ to be $\left(i_{1}, \ldots, i_{k}\right)$-disconjugate for certain values of $i_{1}, \cdots, i_{k}$ without being disconjugate. Our results will provide new information only when our assumptions are weaker than the assumption that $L y=0$ is disconjugate, and the reader is referred to the remarks at the bottom of p. 177 of [9] pointing out some instances when this is not the case. Numerous articles (cf. [10], [11], and [12] and the references therein) have been written establishing relations between different kinds of disconjugacy assumptions.

Sections 2 and 3 deal with two-point and multipoint problems, respectively. In $\S 4$, an example is given which shows how completely the sign of $G(t, s)$ can fail to be determined by $P(t)$ in the absence of any kind of disconjugacy assumption.

We now dispense with some preliminaries. We define the adjoint operator $L^{*}$ in the same way as Hinton [6] and Peterson [9]. That is, define the quasi-derivatives $D_{i}$ and the function classes $A_{i}, i=0, \ldots, n$ recursively by (i) $A_{0}=C[a, b]$ and $D_{0} z=z$ for all $z \in A_{0}$, and (ii) for $1 \leqq k \leqq n, A_{k}=\left\{z \in A_{k-1}: D_{k-1} z \in C^{1}[a, b]\right\}$ and $D_{k} z=\left(D_{k-1} z\right)^{\prime}+$ $(-1)^{k} p_{n-k}(t) z$ for all $z \in A_{k}$. Then $L^{*}$ is defined by $L^{*} z \equiv D_{n} z$. 
Throughout the paper, we let $u_{k}(t, s), 0 \leqq k \leqq n-1$, denote the principal solutions of $L y=0$ at $s$; that is, $u_{k}(t) \equiv u_{k}(t, s)$ is the solution of $L y=0$ satisfying at $s$ the initial conditions $u_{k}^{(l)}(s)=\delta_{k l}$ (Kronecker's delta), $0 \leqq l \leqq n-1$. Also, let $z_{k}(t, s), 0 \leqq k \leqq n-1$, denote the principal solutions of $L^{*} z=0$ at $s$. An important relationship between the principal solutions of $L y=0$ and $L^{*} z=0$ is (see p. 168 of [9])

$$
u_{q}^{(p)}(s, t)=(-1)^{p+q} D_{n-q-1} z_{n-p-1}(t, s),
$$

$p, q=0, \cdots, n-1$ and $s, t \in[a, b]$. In (1.2) as elsewhere in the paper, for a function of two variables, say $f(t, x)$, we denote the $i$ th partial with respect to the first variable by $f^{(i)}(t, x)$ with a similar interpretation for quasi-derivatives.

Let $W\left[f_{1}(t), \cdots, f_{k}(t)\right] \equiv \operatorname{det}\left(f_{j}^{(i-1)}(t)\right), i, j=1, \cdots, k$ denote the "Wronskian" determinant of sufficiently smooth functions $f_{1}, \cdots, f_{k}$. By Cramer's rule, there is a nontrivial solution of $L y=0$ having $(p, n-p)$-zeros at $(\alpha, \beta)$ if and only if

$$
W\left[u_{p}(\beta, \alpha), \cdots, u_{n-1}(\beta, \alpha)\right]=0
$$

wher we intrepret notations such as the left-hand side of (1.3) to be the Wronskian of $u_{p}(t) \equiv u_{p}(t, \alpha), \cdots, u_{n-1}(t) \equiv u_{n-1}(t, \alpha)$ evaluated at $t=\beta$.

When discussing zeros and disconjugacy relative to the adjoint equation $L^{*} z=0$, quasi-derivatives play the same role as ordinary derivatives do for $L y=0$. We will use the fact that $L y=0$ is $(n-p, p)$-disconjugate if and only if $L^{*} z=0$ is $(p, n$-p)-disconjugate (see [6] or [9] for a proof).

2. Two-point problems. The theorem to follow involves the hypotheses:

(H) $L y=0$ is $(p, q)$-disconjugate on $[a, b]$ and $p+q=n$.

$\left(\mathrm{H}_{1}\right) \quad L y=0$ is $(p, 1, q-1)$-disconjugate and $L^{*} z=0$ is $(q, 1$, $p-1)$-disconjugate on $[a, b]$.

$\left(\mathrm{H}_{2}\right) \quad L y=0$ is $(p-1,1, q)$-disconjugate and $L^{*} z=0$ ts $(q-1$, $1, p)$-disconjugate on $[a, b]$.

THEOREM 2.1. If either

(i) $(\mathrm{H}),\left(\mathrm{H}_{1}\right)$ and $1 \leqq q \leqq n-2$,

or

(ii) $(\mathrm{H}),\left(\mathrm{H}_{2}\right)$ and $2 \leqq q \leqq n-1$, holds and $G(t, s)$ is the Green's function for the BVP assigning $(p-q)$ zeros at $(a, b)$, then $P(t)$ determines the sign of $G(t, s)$. 
Proof. Suppose (i) holds. It follows from (H) that

$$
W\left[u_{p}(t, a), \cdots, u_{n-1}(t, a)\right] \neq 0, \quad a<t \leqq b .
$$

We consider the function $g(t, s)$ given by

$$
g(t, s)=\alpha_{p}(s) u_{p}(t, a)+\cdots+\alpha_{n-1}(s) u_{n-1}(t, a), \quad a \leqq t, s \leqq b,
$$

where $\alpha_{p}(s), \ldots, \alpha_{n-1}(s)$ are chosen so that

$$
g^{(i)}(b, s)+u_{n-1}^{(i)}(b, s)=0 \quad(0 \leqq i \leqq q-1)
$$

holds. Such a choice is possible since (2.1) is valid. Then $G(t, s)$ is given by

$$
G(t, s) \equiv \begin{cases}g(t, s), & a \leqq t<s \leqq b \\ g(t, s)+u_{n-1}(t, s), & a \leqq s \leqq t \leqq b\end{cases}
$$

since $G(t, s)$ defined by $(2.3)$ has the properties required of the Green's function.

Now consider the $q$ th order differential operator $M$ defined for $y(t) \in C^{q}(a, b]$ by

$$
M y \equiv W\left[u_{p}(t, a), \cdots, u_{n-1}(t, a), y\right] / W\left[u_{p}(t, a), \cdots, u_{n-1}(t, a)\right] .
$$

The equation $M y=0$ is normal (i.e., has continuous coefficients with the coefficient of the $q$ th order term nonvanishing) on the interval $(a, b]$.

Fix a point $\left(t_{0}, s_{0}\right) \in(a, b) \times(a, b)$. Now $q<n-1$ since (i) holds so $G\left(t, s_{0}\right)$ as a function of $t$ is in $C^{q}(a, b]$ and

$$
M\left[G\left(t, s_{0}\right)\right]= \begin{cases}0, & a<t<s_{0} \\ M\left[u_{n-1}\left(t, s_{0}\right)\right], & s_{0} \leqq t \leqq b .\end{cases}
$$

Since $G\left(t, s_{0}\right) \in C^{q}(a, b)$ has a $q$ th order zero at $t=b$, then

$$
G\left(t, s_{0}\right)=\int_{b}^{t} K(t, \tau) M\left[G\left(\tau, s_{0}\right)\right] d \tau, \quad a<t \leqq b,
$$

where $K(t, \tau)$ is the Cauchy function for the equation $M y=f$. Considering separately the cases $a<t<s_{0}$ and $s_{0} \leqq t \leqq b$ and using the above expression for $M\left[G\left(t, s_{0}\right)\right]$, one obtains the representation

$$
G\left(t, s_{0}\right)= \begin{cases}-\int_{s_{0}}^{b} K(t, \tau) M\left[u_{n-1}\left(\tau, s_{0}\right)\right] d \tau, & a<t<s_{0}, \\ -\int_{t}^{b} K(t, \tau) M\left[u_{n-1}\left(\tau, s_{0}\right)\right] d \tau, & s_{0} \leqq t \leqq b .\end{cases}
$$

For fixed $\tau, K(t, \tau)$ as a function of $t$ is a solution of $M y=0$ with $q-1$ zeros at $t=\tau$. Since $u_{p}(t, a), \cdots, u_{n-1}(t, a)$ form a solution basis 
for $M y=0, K(t, \tau)$ may be extended continuously to $t=a$ and has $p$ zeros at $t=a$. Hence, by the $(p, 1, q-1)$-disconjugacy of $L y=0$,

$$
K(t, \tau) \neq 0, \quad a<t<\tau \leqq b .
$$

We now proceed to show that

$$
M\left[u_{n-1}(\tau, s)\right] \neq 0, \quad a<s<\tau \leqq b .
$$

Let

$$
Z(s, \tau) \equiv M\left[u_{n-1}(\tau, s)\right] W\left[u_{p}(\tau, a), \cdots, u_{n-1}(\tau, a)\right], \quad a<s<\tau \leqq b .
$$

By (1.2), $Z(s, \tau)$ equals the determinant

$$
\left|\begin{array}{cccc}
(-1)^{p} D_{n-1-p} z_{n-1}(a, \tau) & \cdots & (-1)^{n-1} z_{n-1}(a, \tau) & (-1)^{n-1} z_{n-1}(s, \tau) \\
(-1)^{p+1} D_{n-1-p} z_{n-2}(a, \tau) & \cdots & (-1)^{n} z_{n-2}(a, \tau) & (-1)^{n} z_{n-2}(s, \tau) \\
\vdots & & \vdots & \vdots \\
(-1)^{p+q} D_{n-1-p} z_{n-1-q}(a, \tau) & \cdots & (-1)^{n-1+q} z_{n-1-q}(a, \tau) & (-1)^{n-1+q} z_{n-1-q}(s, \tau)
\end{array}\right| .
$$

Note that $Z(s, \tau)$ is a nontrivial linear combination of $z_{p-1}(s, \tau), \cdots$, $z_{n-1}(s, \tau)$ since the $(q, p)$-disconjugacy of $L^{*} z=0$ implies that the coefficient of $z_{p-1}(s, \tau)$ is nonzero. Fix $\tau \in(a, b)$ and let $z(s) \equiv Z(s, \tau)$, $a<s<\tau$. Then $z(s)$, extended continuously to the interval $[a, \tau]$, is a nontrivial solution of $L^{*} z=0$ with $q$ zeros at $s=a$ and $p-1$ zeros at $s=\tau$. Hence, by the $(q, 1, p-1)$-disconjugacy of $L^{*} z=0$, it follows that (2.7) is valid.

We see from (2.5), (2.6), and (2.7) that $G(t, s) \neq 0$ for $(t, s) \in$ $(a, b) \times(a, b)$. To determine the sign of $G(t, s)$, we note that, for $b_{1}$ with $a<b_{1} \leqq b$, the Green's function for the BVP assigning $(p, q)$ zeros at $\left(a, b_{1}\right)$ exists, call it $G\left(t, s, b_{1}\right)$. If $(t, s) \in\left(a, b_{1}\right) \times\left(a, b_{1}\right)$, then (2.5) implies that $\partial G\left(t, s, b_{1}\right) / \partial b_{1}$ is nonzero and has the same sign as $G\left(t, s, b_{1}\right)$; hence, $\left|G\left(t, s, b_{1}\right)\right|$ increases as $b_{1}$ increases. Now pick points $b_{0}$ and $t_{0}$ such that $a<t_{0}<b_{0}<b$ and $L y=0$ is disconjugate on $\left[a, b_{0}\right]$. Then $P\left(t_{0}\right) G\left(t_{0}, t_{0}, b_{0}\right)>0$ since $L y=0$ is disconjugate on $\left[a, b_{0}\right]$; moreover, $\left|G\left(t_{0}, t_{0}, b_{1}\right)\right|$ increases as $b_{1}$ increases from $b_{0}$ to $b$ so $P\left(t_{0}\right) G\left(t_{0}, t_{0}, b\right)>0$. Therefore, $P(t) G(t, s, b)>0$ for all $(t, s) \in$ $(a, b) \times(a, b)$ as desired.

The proof is very similar if (ii) holds. In this case, one lets

$$
\begin{aligned}
g(t, s) & \equiv \beta_{q}(s) u_{q}(t, b)+\cdots+\beta_{n-1}(s) u_{n-1}(t, b) \text { and } \\
G(t, s) & \equiv \begin{cases}g(t, s)-u_{n-1}(t, s), & a \leqq t<s \leqq b \\
g(t, s), & a \leqq s \leqq t \leqq b,\end{cases}
\end{aligned}
$$

and solves for $\beta_{q}(s), \cdots, \beta_{n-1}(s)$. We leave the details to the reader.

REMARK. The proof of Theorem 2.1 actually yields that $|G(t, s)|$ 
increases as the right endpoint increases when (i) holds; that is, if (i) holds and $a<b_{1}<b_{2} \leqq b$ and $(t, s) \in\left(a, b_{1}\right) \times\left(a, b_{1}\right)$, then $\left|G\left(t, s, b_{1}\right)\right|<$ $\left|G\left(t, s, b_{2}\right)\right|$. Similarly, $|G(t, s)|$ can be seen to decrease as the left endpoint increases when (ii) holds. Such monotonicity has been studied by Bates and Gustafson [1] when $L y=0$ is disconjugate.

Theorem 2.1 is essentially Theorem 6 of Peterson [9]. Both draw the same conclusion, but Theorem 2.1 has fewer hypotheses since Peterson assumes $(\mathrm{H}),\left(\mathrm{H}_{1}\right)$ and $\left(\mathrm{H}_{2}\right)$ all hold. The key ideas of considering the Cauchy function for the operator $M$ defined by (2.4) and utilizing (1.2) in the proof are due to Peterson; however, Peterson's proof is considerably more complicated and depends more heavily on the adjoint equation. The greater simplicity is achieved because (2.5) is a simplification of the representation which Peterson obtains; furthermore, it is just this simplification which makes it possible to extend the results to multipoint problems as in the next section.

3. Multipoint problems, As well as $(\mathrm{H})$, we will be interested in the following hypotheses:

$\left(\mathrm{H}_{3}\right) \quad L y=0$ is $(p, 1, \ldots, 1)$-disconjugate or $[a, b]$ (meaning $\left(p, j_{1}, \cdots, j_{n-p}\right)$-disconjugate where $\left.j_{1}=\ldots=j_{n-p}=1\right)$.

$\left(\mathrm{H}_{4}\right) \quad L y=0$ is $(1, \ldots, 1, q)$-disconjugate on $[a, b]$ (that $i s,\left(j_{1}, \cdots\right.$, $\left.j_{n-q}, q\right)$-disconjugate where $j_{1}=\ldots=j_{n-q}=1$ ).

We now state a result due to Peterson [11] which will be instrumental.

Lemma 3.1. Ly=0 is $\left(i_{1}, \cdots, i_{k}\right)$-disconjugate on $[a, b]$ provided that $i_{1}+\cdots+i_{k} \geqq n$ and either

(i ) $(\mathrm{H}),\left(\mathrm{H}_{3}\right)$ and $i_{1} \geqq p$,

or

(ii) $(\mathrm{H}),\left(\mathrm{H}_{4}\right)$ and $i_{k} \geqq q$,

holds.

Under the hypotheses of Lemma 3.1, Green's functions for numerous multipoint problems exist, and we determine the sign in what follows. Lemma 3.2 below is also due to Peterson. For the proof, see Theorem 1 of [11] and Remarks 8, 9, and 10 of [9].

LemmA 3.2. Ly $=0$ is $\left(i_{1}, \cdots, i_{k}\right)$-disconjugate on $[a, b]$ if $i_{1}+$ $\cdots+i_{k} \geqq n$ and either

(i) $i_{1} \geqq p$ and $L y=0$ is $(i, n-i)$-disconjugate on $[a, b], i=$ $p, \cdots, n-1$,

or

(ii) $i_{k} \geqq q$ and $L y=0$ is $(n-i, i)$-disconjugate on $[a, b], i=$ $q, \cdots, n-1$, 
holds. The same statement is true for the adjoint equation $L^{*} z=0$.

We now prove a lemma which supplements the sign information contained in the Levin-Čičkin theorem.

LEMMA 3.3. Suppose $L y=0$ is disconjugate on $[a, b], a<\alpha=$ $a_{1}<\cdots<a_{k}=\beta<b$, and $G(t, s)$ is the Green's function defined on the square $[a, b] \times[a, b]$ for the $\mathrm{BVP}$ assigning $\left(i_{1}, \cdots, i_{k}\right)$-zeros at $\left(a_{1}, \cdots, a_{k}\right)$. Then $P(t) G(t, s) \geqq 0$ on the rectangle $\{(t, s): a \leqq t \leqq b$, $\alpha<s<\beta\}$ with equality only when $t \in\left\{a_{1}, \cdots, a_{k}\right\}, P(t) G(t, s)>0$ on each of the triangles $\{(t, s): a \leqq t<s \leqq \alpha\}$ and $\{(t, s): \beta \leqq s<t \leqq b\}$, and $G(t, s) \equiv 0$ on each of the quadrilaterals $\{(t, s): a \leqq t \leqq b, a \leqq s \leqq$ $\alpha, s \leqq t\}$ and $\{(t, s): a \leqq t \leqq b, \beta \leqq s \leqq b, t \leqq s\}$.

Proof. The argument on pp. 107-108 of [3] given for the square $[\alpha, \beta] \times(\alpha, \beta)$ can be seen to be valid for $(t, s)$ in the rectangle $[a, b] \times(\alpha, \beta)$ so the rectangle conclusion follows. Suppose $s$ is fixed with $a \leqq s \leqq \alpha$. As a function of $t, G(t, s)$ satisfies $L y=0$ and has $n$ zeros on $[s, b]$ from which it follows that $G(t, s) \equiv 0$ for $t \in[s, b]$ and $G(t, s)=-u_{n-1}(t, s)$ for $t \in[a, s]$. Similarly, for $\beta \leqq s \leqq b, G(t, s) \equiv$ 0 for $t \in[a, s]$ and $G(t, s)=u_{n-1}(t, s)$ for $t \in[s, b]$. The rest of the conclusions now follow completing the proof.

We now give the main theorem.

THEOREM 3.1. Suppose $i_{1}+\cdots+i_{k}=n, k \geqq 3$, and either

(i) $(\mathrm{H}),\left(\mathrm{H}_{3}\right), i_{1} \geqq p$ and $L^{*} z=0$ is $(q, 1, p-1)$-disconjugate on $[a, b]$

or $[a, b]$,

(ii) (H), $\left(\mathrm{H}_{4}\right), i_{k} \geqq q$ and $L^{*} z=0$ is $(q-1,1, p)$-disconjugate on holds. If $G(t, s)$ is the Green's function for the BVP assigning $\left(i_{1}, \cdots, i_{k}\right)$-zeros at $\left(a_{1}, \cdots, a_{k}\right)$, then $P(t)$ determines the sign of $G(t, s)$.

Proof. The proof is much like that of Theorem 2.1 with a Green's function for the operator $M$ now playing the role played by the Cauchy function in the proof of Theorem 2.1. Suppose (i) holds. By Lemmas 3.2 and 3.3, it suffices to prove the result when $i_{1}=p$.

Let $g(t, s)$ again be the form (2.2) where $\alpha_{p}(s), \cdots, \alpha_{n-1}(s)$ are now chosen so that

$$
g^{(l)}\left(a_{j}, s\right)+u_{n-1}^{(l)}\left(a_{j}, s\right)=0 \quad\left(2 \leqq j \leqq k, 0 \leqq l \leqq i_{j}-1\right)
$$

holds. $G(t, s)$ is again given by (2.3) and $M$ is again defined by (2.4). 
This time we pick a point $\left(t_{0}, s_{0}\right)$ in $(a, b) \times(a, b)$ and then choose a point $\alpha$ with $a<\alpha<\min \left\{a_{2}, t_{0}, s_{0}\right\}$. The equation $M y=0$ is a normal, $q$ th order, disconjugate equation on the interval $[\alpha, b]$. Let $G_{M}(t, s)$ be the Green's function for the problem

$$
M y=f, y \text { has }\left(i_{2}, \cdots, i_{k}\right) \text {-zeros at }\left(a_{2}, \cdots, a_{k}\right)
$$

on the interval $[\alpha, b]$. $G\left(t, s_{0}\right)$ as a function of $t$ is in $C^{q}[\alpha, b]$ and has $\left(i_{2}, \cdots, i_{k}\right)$-zeros at $\left(a_{2}, \cdots, a_{k}\right)$; hence, we arrive at

$$
G\left(t, s_{0}\right)=\int_{s_{0}}^{b} G_{M}(t, \tau) M\left[u_{n-1}\left(\tau, s_{0}\right)\right] d \tau, \quad \alpha \leqq t \leqq b,
$$

in place of (2.5). The same argument as in Theorem 2.1 shows that (2.7) again holds. It now follows from (3.1), Lemma 3.3, and (2.7) that $G\left(t_{0}, s_{0}\right) \neq 0$ unless $t_{0} \in\left\{a_{2}, \cdots, a_{k}\right\}$.

It remains to be shown that $P(t)$ and $G\left(t_{0}, s_{0}\right)$ are of the same sign when both are nonzero. This will follow from Lemma 3.3 if we can establish that

$$
M\left[u_{n-1}\left(\tau, s_{0}\right]>0, \quad a<s_{0}<\tau<b .\right.
$$

Consider $s_{0}$ fixed and let $w(\tau)$ be defined by

$$
w(\tau) \equiv W\left[u_{p}(\tau, a), \cdots, u_{n-1}(\tau, a), u_{n-1}\left(\tau, s_{0}\right)\right], \quad s_{0} \leqq \tau<b .
$$

The first nonvanishing derivative of $w(\tau)$ at $\tau=s_{0}$ is

$$
w^{(p-1)}\left(s_{0}\right)=W\left[u_{p}\left(s_{0}, a\right), \cdots, u_{n-1}\left(s_{0}, a\right)\right] .
$$

Hence, (3.2) follows from (2.4), (2.7), and (3.3) completing the proof when (i) holds. A similar argument suffices when (ii) holds.

The next theorem follows at once from Theorems 2.1 and 3.1 and Lemma 3.2.

THEOREM 3.2. Suppose $i_{1}+\cdots+i_{k}=n$ and either

(i) $i_{1} \geqq p$ and $L y=0$ is $(i, n-i)$-disconjugate on $[a, b], i=$ $p-1, \cdots, n-1$, or

(ii) $i_{k} \geqq q$ and $L y=0$ is $(n-i, i)$-disconjugate on $[a, b], i=$ $q-1, \cdots, n-1$,

holds. Then $P(t)$ determines the sign of $G(t, s)$ where $G(t, s)$ is the Green's function for the BVP assigning $\left(i_{1}, \cdots, i_{k}\right)$-zeros at $\left(a_{1}, \cdots, a_{k}\right)$.

With exactly the same disconjugacy hypotheses as in Theorems 11 and 12 of [9], Theorem 3.2 shows that $P(t)$ not only determines the sign of two-point problems but determines the sign of multipoint problems as well. 
4. An example. In Remark 7.3 of [4], Gustafson claims that, if $L y=0$ is conjugate on $[a, b]$, then the inequality

$$
G(t, s) / P(t)>0, a<s<b, a \leqq t \leqq b,
$$

may fail at a finite number of points. This seems to indicate that the set of points where (4.1) fails cannot be infinite; however, known results (see Theorems 4 and 5 in [8] and the reference in [8] to earlier Russian literature) show this to be false. It is still of interest to question the location of the points $(t, s)$ in the square $[a, b] \times$ $[a, b]$ where $P(t) G(t, s)<0$. For example, the proofs of Theorems 4 and 5 of [8] produce points close to the line $t=b$ where $P(t) G(t, s)<$ 0 . It is reasonable to question whether all such points must lie outside the square $\left[a, \eta_{1}(a)\right] \times\left[a, \eta_{1}(a)\right]$. Also, in the case of disconjugacy, the zeros of $G(t, s)$ along the line $s=s_{0}$ where $a<s_{0}<b$ are isolated (see Lemma 15, p. 107 of [3]). Perhaps this property still holds even when $L y=0$ is conjugate on $[a, b]$.

We present in this section a third order equation on the interval $[0,4]$ where the Green's function for the BVP assigning $(2,1)$-zeros at $(0,4)$ is zero at all points on the horizontal line segment $\{(t, s)$; $s=1,0 \leqq t \leqq 1\}$ and changes sign on each vertical line crossing this line segment at a point $\left(t_{0}, 1\right)$ with $0<t_{0}<1$. This answers the questions raised above in the negative.

Let $u, v, w, W$ be defined by

$$
\begin{aligned}
& u(t)=1-t, \quad v(t)=t^{2}, \quad w(t)=(t-1)^{2}(4-t), \\
& W(t)=2 t^{3}-6 t^{2}+10 .
\end{aligned}
$$

Then $W(t)$ is the Wronskian of $u(t), v(t), w(t)$ and is nonzero on the interval $[0,4]$; hence, we find $p, q, r \in C[0,4]$, such that $u, v, w$ form a basis for solutions of

$$
L y \equiv y^{\prime \prime \prime}+p(t) y^{\prime \prime}+q(t) y^{\prime}+r(t) y=0, \quad t \in[0,4] .
$$

Any function having a double zero at $t=0$ is a constant multiple of $v(t)$ and $v(4) \neq 0$; hence, the Green's function for the BVP assigning $(2,1)$-zeros at $(0,4)$ exists, call at $G(t, s)$.

We now calculate $\eta_{1}(0)$. Let $z(t)=w(t)-4 u(t)$. Then $z(t)$ has a simple zero at $t=0$ and, since $v(t) \neq 0$ for $t>0$, the results of Sherman [13] show that $\eta_{1}(0)$ is the first zero of $W[v(t), z(t)]$ to the right of $t=0$. A short calculation shows $\eta_{1}(0)=\sqrt{5} / 2$; hence, $\eta_{1}(0)>1$.

The Green's function may be written as

$$
G(t, s)= \begin{cases}(-1 / 16) u_{2}(4, s) t^{2}, & t<s \\ (-1 / 16) u_{2}(4, s) t^{2}+u_{2}(t, s), & t \geqq s .\end{cases}
$$


Let $l$ denote the line segment $l=\{(t, s) ; 0<t<1, s=1\}$. Then $G(t, s)=0$ for all $(t, s)$ on $l$ since $u_{2}(4,1)=0$. We now calculate $\partial G(t, s) / \partial s$ at points on $l$. By using either Peano's formulas (see p. 95 of [5]) or (1.2), one sees that

$$
\frac{\partial}{\partial s}\left[u_{n-1}(t, s)\right]=-u_{n-2}(t, s)+u_{n-1}(t, s) .
$$

Applying this, we see that

$$
\left.\frac{\partial G(t, s)}{\partial s}\right|_{(t, 1)}=\frac{3 t^{2}}{16}, \quad 0 \leqq t<1
$$

Therefore, $G(t, s)$ changes sign on any vertical line passing through $l$.

\section{REFERENCES}

1. P. W. Bates and G. B. Gustafson, Maximization of Green's functions over classes of multipoint boundary value problems, SIAM J. Math. Anal., 7 (1976), 858-871.

2. E. S. Čičkin, A theorem on a differential inequality for multipoint boundary-value prob. lems, Izv. Vysš Ucebn. Zaved. Mat. No. 2 (27), (1962), 170-179.

3. W. A. Coppel, Disconjugacy, Lecture Notes in Mathematics 220, Springer-Verlag, New York, 1971.

4. G. B. Gustafson, A Green's function convergence principle with applications to computation and norm estimates, Rocky Mountain J. Math., 6 (1976), 457-492.

5. P. Hartman, Ordinary Differential Equations, John Wiley, New York, 1964.

6. D. B. Hinton, Disconjugate properties of a system of differential equations, J. Differential Equations, 2 (1966), 420-437.

7. A. Ju. Levin, Non-oscillation of solutions of the equation $x^{(n)}+p_{1}(t) x^{(n-1)}+\cdots+$ $p_{n}(t) x=0$, Russian Math. Surveys, 24 (1969), 43-99.

8. A. C. Peterson, On the sign of the Green's function beyond the interval of disconjugacy, Rocky Mountain J. Math., 3 (1973), 41-51.

9. _- On the sign of Green's functions, J. Differential Equations, 21 (1976) , 167178.

10. On the ordering of multi-point boundary value functions, Canad. Math.

Bull., 13 (1970), 507-513.

11. On a relation between a theorem of Hartman and a theorem of Sherman, Canad. Math. Bull., 16 (1973), 275-281.

12. J. R. Ridenhour, On the zeros of solutions of nth order linear differential equations, J. Differential Equations, 16 (1974), 45-71.

13. T. L. Sherman, Properties of nth order linear differential equations, Pacific J. Math., 15 (1965), 1045-1060.

Received February 26, 1979 and in revised form August 6, 1979. This paper was written while the author was a visiting member of the Department of Mathematics, The University of British Columbia, Vancouver, B. C. V6T 1W5. Support from the National Research Council (Canada) is gratefully acknowledged.

Northern IllinoIs University

DE KALB, IL 60115 


\section{PACIFIC JOURNAL OF MATHEMATICS}

\section{EDITORS}

DONALD BABBITT (Managing Editor)

University of Galifornia

Los Angeles, California 90024

Hugo RossI

University of Utah

Salt Lake City, UT 84112

C. C. MOORE AND ANDREW OGG

University of California

Berkeley, CA 94720
J. DUGUNDJI

Department of Mathematics University of Southern California Los Angeles, California 90007

R. Finn AND J. Milgram Stanford University Stanford, California 94305

\section{ASSOCIATE EDITORS}

R. ARENS

E. F. BECKENBACH

B. H. NEUManN

F. WOLF

K. YOSHIDA

\section{SUPPORTING INSTITUTIONS}

UNIVERSITY OF ARIZONA

UNIVERSITY OF BRITISH COLUMBIA CALIFORNIA INSTITUTE OF TECHNOLOGY

UNIVERSITY OF CALIFORNIA

MONTANA STATE UNIVERSITY

UNIVERSITY OF NEVADA, RENO

NEW MEXICO STATE UNIVERSITY

OREGON STATE UNIVERSITY
UNIVERSITY OF OREGON

UNIVERSITY OF SOUTHERN CALIFONIA

STANFORD UNIVERSITY

UNIVERSITY OF HAWAII

UNIVERSITY OF TOKYO

UNIVERSITY OF UTAH

WASHINGTON STATE UNIVERSITY

UNIVERSITY OF WASHINGTON 


\section{Pacific Journal of Mathematics}

\section{Vol. 92, No. $1 \quad$ January, 1981}

Michael E. Adams and J. Sichler, Lattices with unique complementation . ....1

Walter Allegretto, Positive solutions and spectral properties of second order

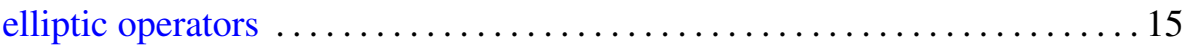

Philip J. Boland and Sean Dineen, Holomorphy on spaces of distribution . . 27

Duncan Alan Buell, Philip A. Leonard and Kenneth S. Williams, Note on

the quadratic character of a quadratic unit $\ldots \ldots \ldots \ldots \ldots \ldots \ldots \ldots \ldots$

Herbert Busemann and Bhalchandra B. Phadke, Two theorems on

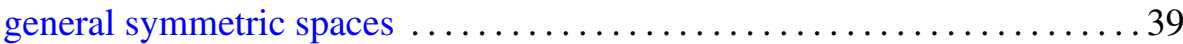

Emeric Deutsch, Bounds for the Perron root of a nonnegative irreducible

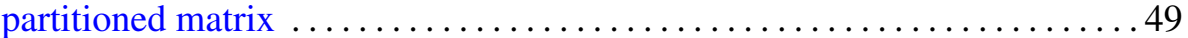

Charles F. Dunkl, A difference equation and Hahn polynomials in two

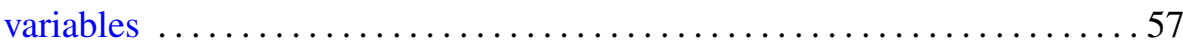

Gustave Adam Efroymson, The Riemann mapping theorem for planar Nash

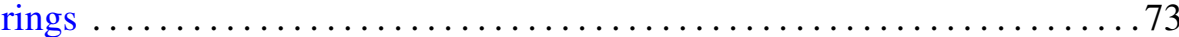

John Albert Fridy and Robert Ellis Powell, Tauberian theorems for matrices generated by analytic functions

Denton Elwood Hewgill, John Hamilton Reeder and Marvin Shinbrot,

Some exact solutions of the nonlinear problem of water waves .......887

Bessie Hershberger Kirkwood and Bernard Robert McDonald, The

symplectic group over a ring with one in its stable range

Esther Portnoy, Transitive groups of isometries on $H^{n}$

Jerry Ridenhour, On the sign of Green's functions for multipoint boundary

value problems

Nina M. Roy, An $M$-ideal characterization of $G$-spaces

Edward Barry Saff and Richard Steven Varga, On incomplete

polynomials. II

Takeyoshi Satō, The equations $\Delta u=P u(P \geqq 0)$ on Riemann surfaces and

isomorphisms between relative Hardy spaces

James Henry Schmerl, Correction to: "Peano models with many generic classes"

Charles Madison Stanton, On the closed ideals in $A(W)$

Viakalathur Shankar Sunder, Unitary equivalence to integral operators

Pavel G. Todorov, New explicit formulas for the $n$th derivative of composite functions

James Li-Ming Wang, Approximation by rational modules on boundary sets

Kenneth S. Williams, The class number of $Q(\sqrt{p})$ modulo 4 , for $p \equiv 5$

$(\bmod 8)$ a prime 